\title{
The Helsinki approach to face transplantation
}

\author{
Lindford, Andrew J.
}

2019-02

Lindford, A J , Mäkisalo , H, Jalanko, H , Lauronen, J , Anttila , V-J , Juteau , S , Ämmälä , A-J , Eskola , A , Saarni , S , Isoniemi , H , Mäkitie , A \& Lassus , P 2019 , ' The Helsinki approach to face transplantation ', Journal of Plastic, Reconstructive \& Aesthetic Surgery , vol. 72 , no. 2 , pp. 173-180 . https://doi.org/10.1016/j.bjps.2018.08.030

http://hdl.handle.net/10138/312540

https://doi.org/10.1016/j.bjps.2018.08.030

publishedVersion

Downloaded from Helda, University of Helsinki institutional repository.

This is an electronic reprint of the original article.

This reprint may differ from the original in pagination and typographic detail.

Please cite the original version. 


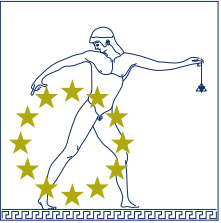

EURAPS

\title{
The Helsinki approach to face transplantation
}

\author{
Andrew J Lindford a,1,*, Heikki Mäkisalob ${ }^{\mathrm{b}}$, Hannu Jalankoc, \\ Jouni Lauronen ${ }^{d}$, Veli-Jukka Anttila ${ }^{e}$, Susanna Juteau ${ }^{f}$, \\ Antti-Jussi Ämmäläg, Anna Eskolag ${ }^{\mathrm{g}}$, Samuli Saarni ${ }^{\mathrm{h}, \mathrm{i}}$, \\ Helena Isoniemi ${ }^{\mathrm{b}}$, Antti Mäkitie ${ }^{\mathrm{j}}$, Patrik Lassus ${ }^{\mathrm{a}, 1}$
}

\author{
a Department of Plastic Surgery, Töölö Hospital, Helsinki University Hospital (HUS), University of \\ Helsinki, Topeliuksenkatu 5, P.O. Box 266, FIN-00029, Helsinki, Finland \\ ${ }^{\mathrm{b}}$ Transplantation and Liver Surgery Clinic, Helsinki University Hospital, University of Helsinki, Finland \\ 'Department of Pediatric Nephrology and Transplantation, Children's Hospital, Helsinki University \\ Hospital, Finland \\ ${ }^{\mathrm{d}}$ Finnish Red Cross Blood Service, Helsinki, Finland \\ e Department of Infectious Diseases, Helsinki University Hospital, University of Helsinki, Finland \\ ${ }^{f}$ Department of Pathology, Haartman Institute, University of Helsinki \& HUSLAB, Helsinki, Finland \\ ${ }^{\mathrm{D}}$ Department of Psychiatry, Helsinki University Hospital, University of Helsinki, Finland \\ h Department of Health, Unit of Mental Health, National Institute for Health and Welfare, Helsinki, \\ Finland \\ 'Turku University Hospital, University of Turku, Turku, Finland \\ ${ }^{j}$ Department of Otorhinolaryngology - Head and Neck Surgery, University of Helsinki, Helsinki University \\ Hospital, Helsinki, Finland
}

Received 11 May 2018; accepted 30 August 2018

\section{KEYWORDS}

Facial transplantation;

Facial

allotransplantation;

VCA program

\begin{abstract}
Aim: We herein describe the establishment of the Helsinki Vascularized Composite Allotransplantation (VCA) program and its execution in the first two face transplant cases. Methods \& patients: The Helsinki VCA program initially required the fulfillment of legal, hospital, financial, and ethical requirements. Thereafter, the assembling of a multidisciplinary team commenced. A team of Plastic, maxillofacial and ENT surgeons comprise the facial VCA team. The protocol involves collaboration with the Solid Organ Transplant (SOT) team, transplant immunology, immunosuppression, microbiology, psychiatric evaluation, well-defined VCA indi-
\end{abstract}

\footnotetext{
${ }^{1} \mathrm{AL}$ and $\mathrm{PL}$ designed and performed the study, collected and analyzed data and wrote the paper. PL was the leader of the program. HM, $\mathrm{HJ}, \mathrm{JL}, \mathrm{V}-\mathrm{JA}, \mathrm{SJ}, \mathrm{A}-\mathrm{JA}, \mathrm{AE}, \mathrm{SS}, \mathrm{HI}$ and $\mathrm{AM}$ all participated in the development of the face transplant program and collected and analyzed data and contributed to writing the paper.

* Corresponding author.

E-mail address: andrew.lindford@hus.fi (A.J. Lindford).
} 
cations and informed consent. Between 2011 and 2017 two patients were selected for transplantation. Both patients had a severe composite facial deformity involving the maxilla and mandible following earlier ballistic injury.

Results: Patient 1 was a 35 year-old male who underwent successful near total face transplantation in February 2016 and at 30 months he has a good aesthetic outcome with symmetrical restoration of the central face and good sensory and symmetrical motor functional outcomes. Patient 2 was a 58 year-old male who underwent full face transplantation in March 2018 and at 5 months he has recovered without major problems.

Conclusion: A successful facial VCA program requires a well-prepared research protocol, experts from multiple specialties and careful patient selection. The establishment of the Helsinki VCA program required long and thorough planning and resulted in the first two Nordic face transplantation cases. This protocol now forms the platform (as a proof of concept) for other types of vascularized composite allotransplantations.

(C) 2018 British Association of Plastic, Reconstructive and Aesthetic Surgeons. Published by Elsevier Ltd. All rights reserved.

\section{Introduction}

Over the past decade we have observed rapid development in the specialty of facial vascularized composite allotransplantation (VCA) with promising functional, aesthetic and psychological outcomes. ${ }^{1}$ At least 39 facial transplantations (including Finland's first two cases) have so far been performed in several centres worldwide. ${ }^{2-21}$ Successful facial VCA demands an effective and coherent multidisciplinary program to be in place. ${ }^{22-24}$ This program should address at the outset the necessary ethical, legal and financial obligations as well as include a well-prepared research protocol, experts from multiple specialties and ultimately, careful patient selection.

The process in establishing the Helsinki VCA program began five years ago and culminated in the first two selected patients successfully undergoing facial allotransplantation in February 2016 and March 2018. We recently reported on the surgical technique and one-year clinical outcome of our first case. ${ }^{25}$ The current paper describes our experience in the setting up of the Helsinki VCA program, details of the protocol and a short description of our first two cases.

\section{Methods and patients}

\section{Permission}

\section{Legal, hospital, financial, and ethical requirements}

The Helsinki Facial VCA program was started in 2011 in the Department of Plastic Surgery, Helsinki University Hospital (HUH). In 2012, vascularized composite allografts were classified as 'organ' transplants in Finland. This facilitated legal permission to be granted by the Ministry of Health as well as the National Supervisory Authority for Social Welfare and Health (Valvira) and the Finnish Medicines Agency (Fimea).

The HUH stipulated a requirement for the VCA program to be aligned with the solid organ transplantation (SOT) team. The main issue was to plan the multiorgan recovery in tandem with the facial allograft recovery in order to ensure the safety of the vital organ recovery. The hospital also required the VCA program to collaborate closely with the Finnish national organ recovery organization. The hospital granted permission only after approval from the other SOT teams.

Ethical issues were given special consideration due to the anticipated public interest and potentially controversial nature of the program. HUH stipulated the requirement for a medical ethicist to assess the ethical implications of the program. Ethical analysis was divided into general issues (evidence in support of this purely experimental treatment, serious risks versus benefits of non-life-saving treatment, autonomy and requirements for truly informed decision making, general issues related to organ transplants) and issues specific for facial transplantation (differences to other, non-visible organ transplants; aesthetic versus medical goals; and most importantly, the potential social reactions to the program and the recipient's perception of these).

The final approval was based on the premise that the program constituted "experimental care". This enabled the program to be financed by the national health care system. The VCA program was subjected to financial evaluation by the health municipality before granting permission to proceed. The cost for the first post-transplant year was estimated to be $150,000 €$. Once approval was obtained, the $\mathrm{HUH}$ gave the final special authorization, and the first patient was placed on the waiting list for facial VCA.

The Helsinki VCA program was also intended to facilitate the future possibility of other VCA's such as hand, laryngeal or abdominal wall transplantations.

\section{Team setup}

\section{Multidisciplinary team setup (Figure 1)}

The Helsinki University Hospital is a tertiary-care academic university hospital with referral area of 1.9 million inhabitants covering one third of the Finnish population. A team of Plastic, maxillofacial and ENT surgeons specializing in head and neck surgery, including 9 surgeons skilled in microsurgery, comprise the Helsinki facial VCA team. Collaboration with the SOT team is of key importance and full access is granted to the multiple subspecialties involved in solid organ transplantation (immunology, infectious diseases, psychiatry, rejection and skin pathology, internal medicine 


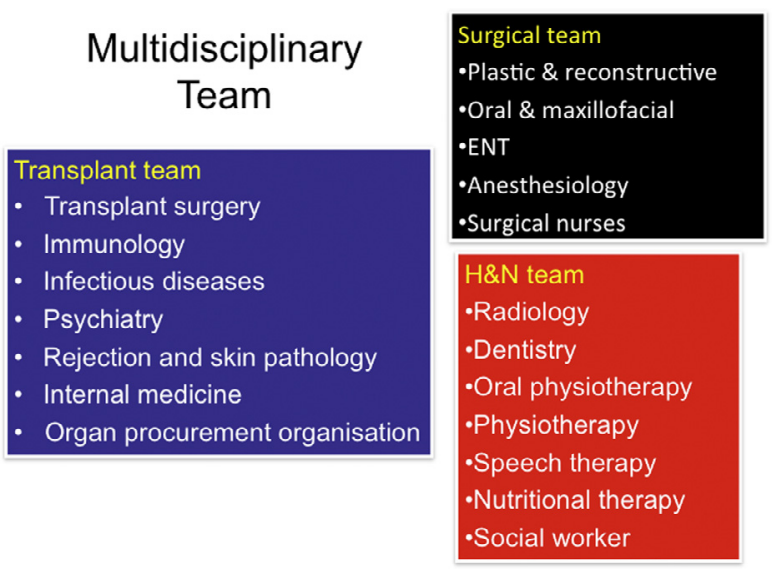

Figure 1 The Helsinki facial VCA team.

and the organ procurement organisation). In addition, the various disciplines (anesthesiology, radiology, dentistry, oral physiotherapy, physiotherapy, speech therapy, nutritional therapy and social services) constituting the Helsinki Head and Neck Cancer Center Service dovetail perfectly with the needs of the VCA program. The team includes dedicated operating room nurses who prepare detailed operation checklists for both the donor and recipient procedures.

\section{Organ transplantation in Finland}

By birth, each person in Finland is a presumed organ donor. In 2016, there were 132 deceased organ donors in Finland (24.7 per million population - the highest number in the Nordic countries), $40 \%$ were over 60 years old, and currently only brain-dead donors are permitted. There is only one transplant centre in Finland in which 399 solid organ transplants were performed (61 transplantations per million of population) in 2016. Donor facial recovery is restricted to the Helsinki region in order to minimize the logistic challenges in spite of the reduction in the donor pool.

\section{Donor consent and organ recovery organization}

A donor consent process and a specialised consent form for facial transplantation was formulated with the SOT team. Consent for facial tissue donation was to be approached only once consent for solid organ donation had been agreed. Furthermore, only haemodynamically stable brain dead organ donors without a significant need for vasoactive inotropic medication, with an accurate cephalometric match, appropriate gender and ethnicity, age $>18$ years and with blood group compatibility were to be considered.

The coordination of the organ recovery operation was of critical importance ( Figure 2). This involved considerable prior planning with the SOT surgeons and simultaneous operating was one of their key demands. Immediate internal core cooling of the donor organs at the time of circulatory arrest is the single most important aspect of any organ preservation technique. This is best achieved by the in situ infusion of a preservation solution chilled to $4^{\circ} \mathrm{C}$, at the time of the circulatory arrest. ${ }^{26}$ In a multiorgan recovery, once all internal organs and the face are ready for removal, cardioplegic and preservation fluids are infused and the donor rapidly cooled. For the face, cold University of

\section{Organ Recovery and Transplant algorithm}

\begin{tabular}{|c|c|}
\hline Organ Recovery algorithm & Recipient patient timeline \\
\hline $\begin{array}{l}\text { Multiorgan recovery } \\
\text { 1. Tracheostomy } \\
\text { 2. Abdominal organ inspection } \\
\text { 2. Simultaneous abdomen and } \\
\text { upper face dissection } \\
\text { 3. Lower face alone (until } \\
\text { osteotomies) } \\
\text { 4. Thoracic organ dissection } \\
\text { 5. Simultaneous perfusion } \\
\text { 6. Thoracic organ detachment } \\
\text { 7. Osteotomies and face } \\
\text { detachment } \\
\text { 8. Abdominal organ detachment }\end{array}$ & $\begin{array}{l}\text { Recipient primary debridement } \\
\text { Recipient definitive debridement }\end{array}$ \\
\hline
\end{tabular}

Figure 2 Organ recovery and transplant algorithm.

Wisconsin solution is infused and this is followed by recovery of the heart and lungs. Recovery of the facial tissues and abdominal organs is then performed.

The recipient patient's debridement commences simultaneously with the donor facial dissection and definitive debridement at the time of donor perfusion.

\section{Donor mask}

The provision of a donor mask was a mandatory requirement stipulated by the $\mathrm{HUH}$; this in spite of open casket burials being quite rare in Finland. A novel additive manufacturing (formerly three-dimensional 3D printing) process was developed and it's various phases tested for the production of a donor face mask that fulfills the requirements for facial restoration after facial recovery. ${ }^{27}$ Data obtained from the processing of a standardized digital image of the donor's face is used for the 3D modeling and printing of a donor face mask.

\section{Cadaveric rehearsals}

Multiple simulated donor face dissections should be rehearsed. We performed these in the Helsinki University Department of Forensic Medicine, Tampere Surgical Education Centre in the Tampere University Hospital and Guy's Campus Anatomy Department at King's College London, UK.

\section{Transplantation immunology}

HLA-typing. The recipient patient is HLA-A, -B and -DR typed. The donor patient is more widely HLA-typed with a Real-Time PCR instrument (Roche Molecular Systems Inc., CA, USA) and Linkage Biosciences HLA-ABCDRDQA1DQB1DP 384 Typing Kit (Linkage Biosciences, CA, USA).

HLA antibodies. The recipient patient's HLA antibodies are determined using Luminex with OneLambda LABScreen ${ }^{\circledR}$ Mixed Class 1 \& 2 and Single Antigen Class I and II kits (OneLambda). A mean fluorecence level (MFI) of 1000 is used as the cut off for a positive finding in single antigen analyses.

Crossmatching. Prior to final transplantation a crossmatch between donor blood T- and B-cells is performed using the complement dependent cytotoxicity method. A negative 
Table 1 Immunological protocol.

\begin{tabular}{ll}
\hline Induction therapy & Maintenance therapy \\
\hline $\begin{array}{l}\text { Pre-op on ward } \\
\text { Mycophenolate mofetil) } 1 \mathrm{~g}, \mathrm{p} .0 .\end{array}$ & $\begin{array}{c}\text { Tacrolimus: } \\
\text { - Trough levels 0-1 month }(13-16 \mathrm{ng} / \mathrm{ml}), 2-6 \mathrm{months}\end{array}$ \\
$\begin{array}{l}\text { Tacrolimus } 0.1 \mathrm{mg} / \mathrm{kg}, \mathrm{p} .0 . \\
(10-15 \mathrm{ng} / \mathrm{ml}),>6 \mathrm{months}(6-8 \mathrm{ng} / \mathrm{ml})\end{array}$ \\
$\begin{array}{l}\text { In theatre } \\
\begin{array}{l}\text { Methylprednisolone } 500 \mathrm{mg}, \text { i.v. } \\
\text { Antithymocyte globulin, ATG } 1,5 \mathrm{mg} / \mathrm{kg}, \text { i.v. (infusion over }\end{array}\end{array}$ \\
$\begin{array}{l}\text { 2-3 hours) and for } 5 \text { days } \\
\text { Methylprednisolone tapering dose }\end{array}$ \\
\hline
\end{tabular}

crossmatch is a requirement for proceeding with the face transplant. In addition, a virtual crossmatch is performed.

\section{Immunosuppression protocol}

The immunosuppression regimen to attempt to prevent rejection includes thymoglobulin as induction therapy and tacrolimus, mycophenolate mofetil, and corticosteroids as maintenance therapy (Table 1).

\section{Rejection diagnostics set-up}

For rejection monitoring, punch biopsies ( $4 \mathrm{~mm}$ in diameter) are taken at recommended intervals and assess rejection according to Banff consensus criteria ${ }^{28,29}$ During the first month biopsies are taken at weekly intervals from the facial graft and sentinel flap. This is followed by monthly biopsies (up to one year) and at 3-monthly intervals thereafter. The biopsies are formalin-fixed and routine hematoxylin-eosin staining performed. At least four sections are performed routinely. For possible further use, slides for immunostainings are also prepared. A sentinel flap has been shown to be a useful tool for rejection monitoring in facial transplantation as it provides a clinical and histological indicator of rejection. It can also be useful in distinguishing a facial dermatitis from a clinical picture of rejection. ${ }^{30}$

\section{Antimicrobial approach}

The patient undergoes serological screening for relevant infectious diseases (Hepatitis A, B, C, E, Cytomegalovirus, Varicella zoster, Epstein-Barr virus, Human herpes virus 6, Human immunodeficiency virus, Syphilis, Tuberculosis, Toxoplasma) and appropriate vaccines are administered (Pneumococci, Meningococci, Haemophilus influenzae type $B$, hepatitis A,B, Diphtheria, Tetanus, seasonal influenza and chicken pox if the patient is not naturally immunized).

In Epstein-Barr virus (EBV) negative recipients EBV positive donors are contraindicated. With regard to cytomegalovirus (CMV) prophylaxis, the patient receives acyclovir for 3 months (recipient (R) negative / donor (D) negative), valganciclovir for 6 months (R+/D+ or $R+/ D-)$, and valganciclovir for 12 months $(R-/ D+)$. Co-trimoxazole is given for at least 6 months as a prophylactic regimen against Toxoplasma gondii and Pneumocystis jirovecii. Perioperatively, antibiotic regimens are adjusted according to the results of cultures from both the donor and recipient. Antifungal prophylaxis using anidulafungin is given after transplantation for one week.

\section{Psychiatric evaluation protocol}

A preoperative evaluation aims to ensure that a candidate has sufficient psychological and social resources to cope with the transplantation process and be compliant with lifelong immunosuppressive medication, rehabilitation, and follow up. ${ }^{31-33}$ The patient's ability to receive and process information is assessed in order to evaluate capability to give informed consent. ${ }^{34} \mathrm{~A}$ semi-structured interview by a clinical psychologist takes place on at least 3 occasions and aims to evaluate the candidate's readiness and preparedness for the operation and to predict psychological reactions, possible risk factors and compliance with treatment after transplantation. The extent to which cognitive tests are used varies as some of the candidates may have preexisting brain damage or organic brain illness.

A plan must also be in place for those candidates that are deemed not to be a suitable transplantation candidate, since these patients also need support and follow up.

Finally, the VCA team must plan the postoperative follow-up and support schedule. During the early postoperative period, assessment by a psychiatrist or psychologist should be available once a week and following hospital discharge every 1-2 months. In addition, every 6 months BDI (Beck Depression Inventory), OASIS (Overall Anxiety Severity and Impairment Scale), and AUDIT (Alcohol Use Disorders Identification Test) questionnaires should be completed.

\section{Indications for facial transplantation}

We define the indications to include a functional facial deficiency that cannot be reconstructed with conventional techniques including functional loss of the oral and/or periocular sphincters and/or significant loss of facial structures in general. Absolute contraindications would include predicted non-compliance, recent history $(<5$ years) of malignancy, psychiatric liability or severe chronic disease. However, the patient's perspective is of fundamental importance with evidence of a significantly poor quality of life a chief determinant of suitability for facial VCA.

\section{Informed patient consent}

We ensure that the patients will be fully informed about all the risks, post-operative course and possible sequelae. Ultimately, it is the patient's decision.

\section{Patients}

The population of Finland is $\mathbf{5 . 5}$ million and there are five university hospitals that treat the most severe facial trauma patients. Between 2011 and 2017 the Helsinki facial VCA team has evaluated six patients. These patients have severe facial aesthetic and functional deformities resulting from 
ballistic injuries $(n=3)$, burns $(n=2)$ and neurofibromatosis $(n=1)$.

Functional assessment involved the analysis of: facial anatomical region affected, loss of facial function (breathing, mastication, swallowing, speaking, labial competence and eyelid closure), aesthetic defect, motor and sensory function. Motor function was assessed using the Sunnybrook Facial Grading System. Sensory function was assessed using the Semmes Weinstein monofilament test for light touch and two-point discrimination. Quality of life (QOL) was assessed using the generic $15 \mathrm{D}$ health related $\mathrm{QOL}$ instrument and the European Organization for Research and Treatment of Cancer QOL Questionnaire (EORTC QLQ-Head \& Neck 35 module).

Two patients with severe soft tissue and bony midface deformities following earlier ballistic trauma were selected for facial transplantation.

Patient 1 was a 35 -year old blind male with a severe central facial deformity involving maxilla and mandible and including loss of facial height. He had severe symptoms related to nasal breathing, eating, speech, and recurrent soft tissue infections.

Patient 2 was a 59-year old male with a severe full facial deformity also with involvement of maxilla and mandible. He had a permanent tracheostomy and problems with lip competence, eating, speech, and left eye dryness due to insufficient lid closure.

There are two further patients in Finland currently under consideration for facial VCA, one patient following burn injury and the other following ballistic trauma.

\section{Results}

\section{Patient 1}

\section{Surgical procedure}

Patient 1 was on the waiting list for two months prior to transplantation. He underwent successful face transplantation in February 2016. The facial transplant consisted of a Le Fort II maxilla, central mandible, lower $2 / 3$ of the midface muscles, facial and neck skin, oral mucosa, anterior tongue and floor of mouth muscles, facial nerve ( 3 bilateral branches), and bilateral hypoglossal and buccal nerves. A sentinel flap (radial forearm flap) recovered from the donor was used to provide skin biopsies for monitoring of rejection.

Donor facial allograft retrieval duration was 6 hours (total multiorgan recovery duration was 10 hours), the duration of the recipient debridement was 10 hours and final facial restoration 9 hours. The facial recovery was performed simultaneously with the SOT recovery according to the agreed algorithm with no significant deviations (Figure 2).

\section{Immunological and medical outcome}

Pre-transplantation results. The donor had 4 mismatching HLA-antigens $\left(A^{*} 03, B^{*} 07, D R B 1 * 13, D R B 1 * 15\right)$. Cytotoxic crossmatches between the donor and recipient were negative for both T- and B-cells. The patient had preoperatively developed antibodies against B7 HLA antigen (MFI $1967)$, thus virtual crossmatch was positive. This finding was considered an increased immunological risk but not an absolute contraindication.

Post-transplantation results. At 30 months follow-up there have thus far been no episodes of acute rejection and all biopsies taken from the facial allograft and sentinel flap have been Banff grade 0. Because of a fear of acute and potential chronic rejection we elected against the 'minimisation' of immunosuppression.

The patient developed a nasopalatinal fistula on the seventh postoperative day and required three surgical attempts until closure at 14 months. Plasma creatinine increased two months after the operation and has decreased to $1.2 \mathrm{mg} / \mathrm{dl}$ at 24 months. The patient suffered transient diabetes during the initial period due to high doses of methylprednisolone (up to 3 months postoperatively). The development of hypertension has also required medical therapy.

Infection results. The donor was $\mathrm{CMV}$ and toxoplasma negative but EBV positive. The recipient was both CMV and EBV positive, and toxoplasma negative. CMV-PCR has been negative during the whole follow-up but EBV-PCR has been twice slightly over the detection level. The patient has not had any other major bacterial or fungal infections.

\section{0-month functional outcome}

The patient has undergone intense physio-, occupational-, and speech therapy. Follow up has also included regular dental and ENT assessments. Functional assessments included sensory evaluation (light touch, pain, 2 point discrimination) at 1, 2, 3, 4, 5, 6, 9, 12, 18, 24 and 30 months. Motor assessment involved manual muscle testing of facial expression at similar time intervals and also included the use of the Sunnybrook Facial Grading System and Electromyography at 6,12 and 24 months.

At 30 months, the patient has a good aesthetic outcome with symmetrical restoration of the mobile central part of the face with recovery of pain and light touch sensation to the entire facial skin and intraoral mucosa and with symmetrical muscle activity in the floor of the mouth and facial musculature and the patient is able to produce spontaneous smile.

\section{Psychological results}

Prior to transplantation, the patient exhibited a good and stable mental state with no psychiatric, psychological, or substance abuse problems diagnosed. Following transplantation regular contact with either the psychiatrist or psychologist has been provided but no medication has been needed and his mental state has remained stable. At 24 months postoperatively, the patient completed four questionnaires: BDI, OASIS, Audit, and 15D. The results did not suggest a risk of depression, anxiety or alcohol abuse. Successful social and psychological outcomes have also been observed with improvement in some QOL domains (15D, EORTC H\&N-35). These QOL questionnaires were completed at 6,12 and 24 months post-operatively. 


\section{Patient 2}

\section{Surgical procedure}

Patient 2 awaited a donor for 15 months until successful transplantation in March 2018. The transplant included full-face soft tissues, Le Fort II maxilla, central mandible, oral mucosa, floor of mouth muscles, bilaterally 4 branches of the facial and hypoglossal nerves and bilateral sensory nerves (supraorbital, infraorbital, buccal and mental). A sentinel flap (radial forearm flap) was again recovered from the donor to provide skin biopsies for monitoring of rejection.

Donor facial allograft recovery duration was 6 hours (total multiorgan recovery duration was $11 \mathrm{~h}$ ), the duration of the recipient debridement was $12 \mathrm{~h}$ and final facial restoration $14 \mathrm{~h}$. The facial recovery was again performed simultaneously with the SOT recovery according to the agreed algorithm with no significant deviations (Figure 2 ).

\section{Immunological and early medical outcome}

\section{Post-transplantation results}

Cytotoxic crossmatches between the donor and recipient were negative for both $\mathrm{T}$ - and B-cells. The patient did not present any preformed donor specific antibodies in the virtual cross match.

The patient spent 10 days on ICU and 6 weeks on the ward. Of the postoperative problems, the patient developed a unilateral parotid sialocele, and a partial necrosis of the hard palate soft tissues and a small oronasal fistula. The sialocele was treated successfully with botulinum toxin and the palatinal necrosis was revised and fistula closed surgically. There has not been any medical complication or immunosuppressive medication related side effects so far. At 5 months, he has developed partial facial graft sensation and slight movements of the transplanted orbicularis oris, zygomatic and orbicularis oculi muscles.

\section{Financial cost}

The financial cost of the operation was approximately 75,000 euros for patient 1 and the overall cost for the first year after transplantation was approximately 190,000 euros.

\section{Discussion}

The 5-year process in the setting-up of the Helsinki VCA program required thorough planning and painstaking attention to detail and resulted in successful face transplantation in two patients in 2016 and 2018. At the earliest stage, an open social dialogue was conducted consisting of an open seminar for professionals and the press, prior to the start of the program. This included analysis of the ethical issues to help inform public debate. The planning phase received large and overwhelmingly positive Finnish media coverage. Subsequently, the legal, hospital, and financial requirements were each addressed before final permission to proceed was granted by the hospital authorities.
The formation of the multidisciplinary team then began in earnest led by the Head of the HUH Head \& Neck Plastic surgery team (PL). A clinical protocol was compiled and drawing on the appropriate sub disciplines of the Helsinki Head \& Neck Cancer Centre and Helsinki SOT service, a wide-ranging and well-equipped Multidisciplinary Facial VCA team was assembled. In particular, the immunological and infection control aspects demanded careful analysis and planning. This was in view of the fact that these elements have to date been implicated in nearly all cases of facial VCA transplant patient morbidity and or mortality and constitutes the major overall risk of the procedure. In addition, the post-operative rehabilitation protocol warranted clear and focused multidisciplinary planning to meet the multiple challenges likely to be encountered during the recovery period.

The indications for facial VCA were clearly defined as those central facial anatomical defects unable to be satisfactorily reconstructed by conventional means. However, the focus would be the patient and a clear potential benefit to his/her quality of life was essential for inclusion into the VCA program. The psychological assessment was of fundamental importance in ensuring that a selected patient had the required resources to cope with the magnitude of the operation and life-long immunosuppression. Furthermore, our two selected patients are both victims of self-inflicted ballistic trauma and hence effective psychiatric screening and confirmation of a strong social network are pertinent. Indeed, both patients have remained psychologically stable throughout the peri-operative and post-operative period with improvement in several quality of life domains in the first patient. Of note, our first patient has complete bilateral blindness but a previous position paper had concluded that blind patients could be included in facial VCA programs on the basis of functional, social, rehabilitative and ethical grounds. ${ }^{35}$

During the five-year developmental process and establishment of the Helsinki Facial VCA programme considerable planning was involved concerning the logistics and coordination of the face transplant operation. The importance of a team approach cannot be overstated. In particular the synchronous procurement protocol needs to be carefully planned with the SOT surgeons. ${ }^{36-40}$ In our institution the facial procurement occurs in a physically separate hospital site to the recipient operation. This presented its own challenges with the continuous and effective, dynamic communication between both the procurement and recipient teams essential for success. Furthermore, the duration of the recipient debridement operation was longer than the donor face harvest. This was anticipated yet still presented a difficulty in planning the synchronization of both operations. The recipient debridement cannot be commenced too early in case the donor allograft fails or problems are encountered during the multiorgan recovery operation. Conversely, if the donor facial allograft is detached too soon, the problem of increasing ischaemia time would be encountered. Thus, we had to define the point of no return and initiate definitive debridement at a stage when the transplant feasibility was certain (Figure 2 ). Thus, cadaver practicing was essential for the planning of the harvesting algorithm as well as for the timing of the donor mask manufacturing. 
Our first two patients underwent successful facial transplantation and their data have been submitted to the International Registry for Hand and Composite Tissue Allotransplantation (IRHCTT). With regards to patient 1, although the patient has encountered various postoperative challenges including infectious episodes, immunosuppression related side-effects and a need for surgical corrections; all of these challenges have been successfully managed by the multidisciplinary team. ${ }^{25}$

Pertinently, we have not observed any clinical nor histological signs of acute rejection (at 30 months follow-up). This is in spite of the presence of a 4/6 HLA mismatch and initial donor specific Class-I HLA antibody at the time of transplantation. The absence of rejection may in part be accounted for by appropriate patient selection and compliance as well as close continuous physician contact during the rehabilitation period. Non-compliance is a major risk factor for acute rejection and graft failure of renal transplants. ${ }^{41,42}$ Hence, the patient is likely to benefit from close, regular, and long-term contact in order to preserve motivation for compliance as well as for successful functional facial outcome. More importantly, various aspects of speaking, swallowing and breathing should be covered by the rehabilitation phase in the programme. Therefore, the team should include a holistic plan for long-term follow-up with similar intensity and emphasis on psychosocial factors.

The actual cost for the transplantation exceeded the estimated budget by $25 \%$. Most of the extra costs were related to additional unplanned but necessary corrective surgical procedures. All the VCA program costs in Finland are covered by the state, since the program belongs to the National Organ Transplant program. This is in contrast with many other countries where active centers or programs at an early set-up phase are suffering from financial difficulties due to reliance on non-state sponsors. ${ }^{43}$ Therefore, secure state sponsorship is beneficial for a long-term survival of a VCA program. Furthermore, there is a need for more objective measurements of pre- and post-transplant results. Current data in the literature are somewhat lacking in describing the true value that face transplantation provides. Current QOL measures are not effective in measuring the improvements specific to the function of the face. The Cleveland Clinic has previously proposed the FACES Score ${ }^{44}$, and more recently the Face-Q (a patient reported outcome tool for head and neck reconstruction) has also been described. ${ }^{45}$ The latter could potentially be adapted for the face transplant population.

The issue of population size in relation to the provision of a facial VCA program needs further consideration. Our second patient had been on the waiting list for 15 months in spite of three suitable donors. Whilst consent was obtained for solid organ procurement the next of kin declined the donation of the facial tissues. This underlines the need for a large donor catchment area especially for preimmunized patients for whom suitable donors are more scarce. In addition, a requirement for an active facial VCA center should be an adequate number of selected patients for transplantation. Thus, Finland with its small population size, raises the question for the future regarding possible centralization of our VCA program to serve the Northern European region. One argument to support this view is the fact that all the five Nordic countries share a rather similar public health-care system, which is regulated by governmental authorities.

In conclusion, we described the long and thorough process involving numerous medical disciplines in establishing the Helsinki Facial VCA protocol and resulting in the first two successful Northern European facial transplantation cases in 2016 and 2018.

\section{Conflict of interest statement}

The authors report no conflict of interest.

\section{Acknowledgements}

University of London, Department of Anatomy, Hodgkin Building, Guy's Campus, London, SE1 1UL.

Department of Pathology, Helsinki University Hospital, Helsinki, Finland.

A special thanks to Bohdan Pomahac, Elof Eriksson and Julian Pribaz of the Brigham and Women's Hospital, Boston, USA for their assistance in the setting up of the Helsinki Facial VCA program.

\section{References}

1. Khalifian S, Brazio PS, Mohan R, et al. Facial transplantation: the first 9 years. Lancet 2014;384(9960):2153-63.

2. Sosin M, Rodriguez ED. The face transplantation update: 2016. Plast Reconstr Surg. 2016;137(6):1841-50.

3. Devauchelle $B$, Badet $L$, Lengelé $B$, et al. First human face allograft: early report. Lancet 2006;368(9531):203-9.

4. Guo S, Han Y, Zhang X, et al. Human facial allotransplantation: a 2-year follow-up study. Lancet 2008;372(9639):631-8.

5. Siemionow M, Papay F, Alam D, et al. Near-total human face transplantation for a severely disfigured patient in the USA. Lancet 2009;374(9685):203-9.

6. Lantieri L, Hivelin M, Audard V, et al. Feasibility, reproducibility, risks and benefits of face transplantation: a prospective study of outcomes. Am J Transpl 2011;11(2):367-78.

7. Lantieri L, Grimbert P, Ortonne N, et al. Face transplant: long-term follow-up and results of a prospective open study. Lancet 2016;388(10052):1398-407.

8. Pomahac B, Pribaz J, Eriksson E, et al. Restoration of facial form and function after severe disfigurement from burn injury by a composite facial allograft. Am J Transpl 2011;11(2):386-93.

9. Pomahac B, Pribaz J, Eriksson E, et al. Three patients with full facial transplantation. N Engl J Med 2012;366(8):715-22.

10. Cavadas PC, Ibáñez J, Thione A. Surgical aspects of a lower face, mandible, and tongue allotransplantation. J Reconstr Microsurg 2012;28(1):43-7.

11. Petruzzo P, Kanitakis J, Testelin S, et al. Clinicopathological findings of chronic rejection in a face grafted patient. Transplantation 2015;99(12):2644-50.

12. Sicilia-Castro D, Gomez-Cia T, Infante-Cossio P, et al. Reconstruction of a severe facial defect by allotransplantation in neurofibromatosis type 1: a case report. Transpl Proc 2011;43(7):2831-7.

13. Barret JP, Gavaldà J, Bueno J, et al. Full face transplant: the first case report. Ann Surg 2011;254(2):252-6.

14. Lantieri L. Face transplant: a paradigm change in facial reconstruction. J Craniofac Surg 2012;23(1):250-3.

15. Roche NA, Vermeersch HF, Stillaert FB, et al. Complex facial reconstruction by vascularized composite allotransplan- 
tation: the first Belgian case. J Plast Reconstr Aesthet Surg 2015;68(3):362-71.

16. Özel AȘ, Güçlü ZA, Gülșen A, Özmen S. The Importance of the condition of the donor teeth and jaws during allogeneic face transplantation. J Craniofac Surg 2015;26(4):1338-41.

17. Dorafshar AH, Bojovic B, Christy MR, et al. Total face, double jaw, and tongue transplantation: an evolutionary concept. Plast Reconstr Surg 2013;131(2):241-51.

18. Chandraker A, Arscott R, Murphy GF, et al. The management of antibody-mediated rejection in the first presensitized recipient of a full-face allotransplant. Am J Transplant 2014;14(6):1446-52.

19. Maciejewski A, Krakowczyk Ł, Szymczyk C, et al. The first immediate face transplant in the world. Ann Surg 2016;263(3):e36-9.

20. Westvik TS, Dermietzel A, Pomahac B. Facial restoration by transplantation: the Brigham and Women's face transplant experience. Ann Plast Surg 2015;74(Suppl 1):S2-8.

21. Sosin M, Ceradini DJ, Levine JP, et al. Total face, eyelids, ears, scalp, and skeletal subunit transplant: a reconstructive solution for the full face and total scalp burn. Plast Reconstr Surg 2016;138(1):205-19.

22. Pomahac B. Establishing a composite tissue allotransplantation program. J Reconstr Microsurg 2012;28(1):3-6.

23. Siemionow MZ, Gordon CR. Institutional review board-based recommendations for medical institutions pursuing protocol approval for facial transplantation. Plast Reconstr Surg 2010;126(4):1232-9.

24. Siemionow M, Gordon CR. Overview of guidelines for establishing a face transplant program: a work in progress. Am J Transpl 2010;10(5):1290-6.

25. Patrik Lassus, Andrew Lindford, Jyrki Vuola, et al. The Helsinki face transplantation: surgical aspects and one-year outcome. $J$ Plast Reconstr Aesthet Surg 2018;71(2):132-9.

26. Belzer FO, Southard JH. Principles of solid-organ preservation by cold storage. Transplantation 1988;45(4):673-6.

27. Mäkitie AA, Salmi M, Lindford A, Tuomi J, Lassus P. Three-dimensional printing for restoration of the donor face: a new digital technique tested and used in the first facial allotransplantation patient in Finland. J Plast Reconstr Aesthet Surg 2016;69(12):1648-52.

28. Hautz T, Zelger BG, Weissenbacher A, et al. Standardizing skin biopsy sampling to assess rejection in vascularized composite allotransplantation. Clin Transplant 2013;27:81-90.

29. Cendales LC, Kanitakis J, Schneeberger S, et al. The Banff 2007 working classification of skin-containing composite tissue allograft pathology. Am J Transpl 2008;8:1396-400.

30. Kueckelhaus M, Fischer S, Lian CG, et al. Utility of sentinel flaps in assessing facial allograft rejection. Plast Reconstr Surg 2015; 135(1):250-8.
31. Edgerton MT, Langman MW, Pruzinsky T. Plastic surgery and psychotherapy in the treatment of 100 psychologically disturbed patients. Plast Reconstr Surg 1991;88(4):594-608.

32. Furr LA, Wiggins O, Cunningham M, et al. Psychosocial implications of disfigurement and the future of human face transplantation. Plast Reconstr Surg 2007;120(2):559-65.

33. Dew MA, DiMartini AF, Steel J, et al. Meta-analysis of risk for relapse to substance use after transplantation of the liver or other solid organs. Liver Transpl 2008;14(2):159-72.

34. Bunzel B, Laederach-Hofmann K. Solid organ transplantation: are there predictors for posttransplant noncompliance? A literature overview. Transpl 2000 15;70(5):711-16 Review.

35. Carty MJ, Bueno EM, Lehmann LS, Pomahac B. A position paper in support of face transplantation in the blind. Plast Reconstr Surg 2012;130(2):319-24.

36. Bueno EM, Diaz-Siso JR, Pomahac B. A multidisciplinary protocol for face transplantation at Brigham and Women's Hospital. J Plast Reconstr Aesthet Surg 2011;64(12):1572-9.

37. Barret JP, Serracanta J, Collado JM, et al. Full face transplantation organization, development, and results - the Barcelona experience: a case report. Transpl Proc 2011;43(9):3533-4.

38. Bueno J, Barret JP, Serracanta J, et al. Logistics and strategy of multiorgan procurement involving total face allograft. Am J Transpl 2011;11(5):1091-7.

39. Meningaud JP, Benjoar MD, Hivelin M, Hermeziu O, Toure G, Lantieri L. Procurement of total human face graft for allotransplantation: a preclinical study and the first clinical case. Plast Reconstr Surg 2010;126(4):1181-90.

40. Brazio PS, Barth RN, Bojovic B, et al. Algorithm for total face and multiorgan procurement from a brain-dead donor. Am J Transpl 2013;13(10):2743-9.

41. Lerut E, Kuypers DR, Verbeken E, et al. Acute rejection in noncompliant renal allograft recipients: a distinct morphology. Clin Transpl 2007;21(3):344-51.

42. Gaynor JJ, Ciancio G, Guerra G, et al. Graft failure due to noncompliance among 628 kidney transplant recipients with long-term follow-up: a single-center observational study. Transplantation 2014;97(9):925-33.

43. Proceedings of the 13 th congress of the international society of vascularized composite allotransplantation, Salzburg, Austria; 2017 October 26th-27th.

44. Gordon CR, Siemionow MZ, Coffman KL, et al. The Cleveland Clinic FACES Score: a preliminary assessment tool for identifying the optimal face transplant candidate. J Craniofac Surg 2009;20:1969-74.

45. Pusic AL, Klassen AF, Scott AM, Cano SJ. Development and psychometric evaluation of the FACE-Q satisfaction with appearance scale: a new patient-reported outcome instrument for facial aesthetics patients. Clin Plast Surg 2013;40(2):249260. 